\title{
Non-invasive determination of cardiac output by Doppler echocardiography and electrical bioimpedance
}

\author{
David B Northridge, Iain N Findlay, John Wilson, Esther Henderson, Henry J Dargie
}

\begin{abstract}
Cardiac output measured by thermodilution in 25 patients within 24 hours of acute myocardial infarction was compared with cardiac output measured by Doppler echocardiography (24 patients) and electrical bioimpedance ( 25 patients). The mean (range) cardiac outputs measured by Doppler $(4 \cdot 03(2 \cdot 2-6 \cdot 0) 1 / \mathrm{min})$ and electrical bioimpedance $(3 \cdot 79(1 \cdot 1-6 \cdot 2) 1 / \mathrm{min})$ were similar to the mean thermodilution value (3.95 (2.1-6.2) $1 / \mathrm{min})$. Both noninvasive techniques agreed closely with thermodilution in most patients. None the less, three results with each method disagreed with thermodilution by more than $11 / \mathrm{min}$.

Both non-invasive techniques were reproducible and accurate in most patients with acute myocardial infarction. Doppler echocardiography was time consuming and technically demanding. Electrical bioimpedance was simple to use and had the additional advantage of allowing continuous monitoring of the cardiac output.
\end{abstract}

Knowledge of the cardiac output is valuable in the management of patients with acute myocardial infarction because it identifies patients at greatly increased risk and also provides a rapid means of monitoring the response to treatment. ${ }^{1}$ Most patients, however, are managed without measurement of cardiac output-by relying on clinical signs. Unfortunately these can be misleading in this group of patients. Arterial blood pressure may be maintained in the face of a low cardiac output by raised systemic vascular resistance. A fall in urine output is an indication of reduced renal blood flow, but this may be due to activation of the sympathetic nervous system and does not necessarily indicate a low cardiac output. ${ }^{2}$ The chest $x$ ray is a sensitive indicator of raised left atrial pressure, ${ }^{2}$ but measurement of cardiac output has until recently required right heart catheterisation. An accurate non-invasive method of measuring cardiac output would therefore have many attractions: two of the most promising techniques are Doppler echocardiography and electrical bioimpedance.

Although a high degree of accuracy has been claimed for both methods, ${ }^{3-7}$ all of these studies were conducted in normal volunteers subjected to various artificial means of altering cardiac output or in patients in intensive care with a wide range of haemodynamic disturbances.
None of the previous studies of electrical bioimpedance and only one study of Doppler included patients with acute myocardial infarction. $^{3}$

A new bioimpedance monitor (BoMed NCCOM3) has recently been developed that simplifies the measurement of cardiac output. We wished to compare the accuracy of this new monitor with Doppler echocardiography in acute myocardial infarction. It is not possible to extrapolate from results obtained in normal volunteers during exercise or patients in general intensive care because patients with myocardial infarction may have much lower cardiac outputs.

The aims of this study were to assess the feasibility, reproducibility, and ease of use of both non-invasive techniques in patients with recent ( $<24$ hours) myocardial infarction and to compare cardiac output determined by Doppler echocardiography and electrical bioimpedance with that obtained by thermodilution in the same patients.

\section{Patients and methods}

PATIENTS

We studied men and women aged $<75$ years admitted to our coronary care unit with a diagnosis of acute myocardial infarction. All patients were studied within 24 hours of the onset of chest pain. Patients were included in the study if there was a clinical indication for Swan-Ganz catheterisation (early heart failure, hypotension, or poor urine output).

Patients were excluded if they had any condition known to interfere with the bioimpedance signal such as obesity or pleural effusion. An initial screening echocardiographic study was performed to exclude patients with significant aortic valve disease, tricuspid incompetence, or pericardial effusion.

\section{SWAN-GANZ CATHETERISATION}

A 7F, triple lumen, Edwards Laboratories Swan-Ganz catheter was advanced from an antecubital vein to the pulmonary artery under fluoroscopic control. Intracardiac and pulmonary artery pressures were recorded and patients were then returned to coronary care. Thermodilution cardiac output was measured by an Edwards Laboratories cardiac output computer with $10 \mathrm{ml}$ iced dextrose. The mean of three injections, all within $10 \%$ of each other and excluding the first injection, was recorded. It was not possible to perform all three techniques simultaneously because the bioimpedance monitor interferes with the Doppler signal. Therefore, Doppler recordings of aortic 
Figure 1

Echocardiographic measurement of the diameter of the left ventricular outflow tract. Ao, aorta; $L A$, left atrium; $L V$, left ventricle, $R V$, right ventricle.

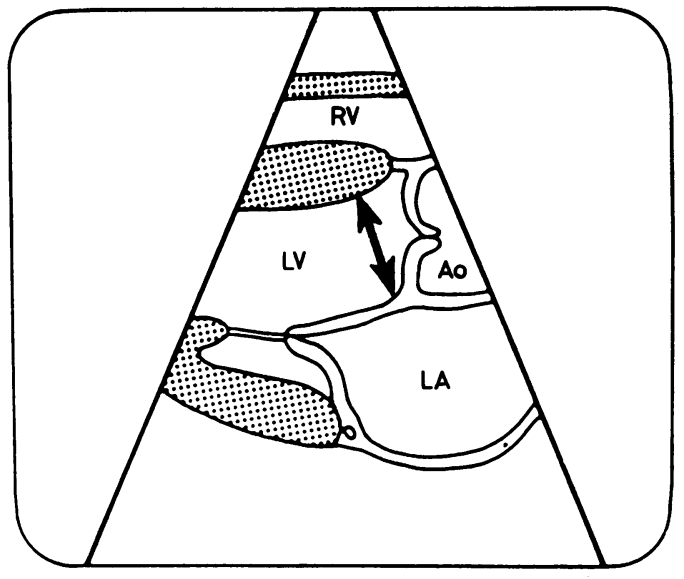

flow were recorded immediately before or immediately after the other two techniques.

DOPPLER ECHOCARDIOGRAPHY

The diameter of the left ventricular outflow tract was used to calculate the cross sectional area, assuming a circular profile. Measurements were made by cross sectional echocardiography in the parasternal long axis view (Hewlett Packard model 77020A with a 2.5 $\mathrm{MHz}$ phased array transducer). With the image frozen in midsystole, the diameter of the left ventricular outflow tract was measured immediately proximal to the aortic valve leaflets as described by Lewis et al. ${ }^{5} \mathrm{We}$ calculated the diameter as the mean of five measurements using the trailing-edge to leading-edge method (fig 1).

We measured aortic blood velocity by continuous wave Doppler ultrasound using a small $1.9 \mathrm{MHz}$ probe and the same Hewlett Packard system. Care was taken to obtain the clearest spectral profile from both the suprasternal and apical windows. When both windows were successful the site giving the highest aortic flow velocities was used for analysis, as described by Bouchard et al. ${ }^{6}$ No attempt was made to measure and correct for the angle between the continuous wave Doppler ultrasound beam and the direction of blood flow. We took hard copies on a strip chart recorder and analysed them off line using a Kontron computer and magnetised digitising tablet. Stroke distance was taken as the mean of 10 measurements of the area under the time velocity curve. Doppler cardiac output was calculated as:

$$
\mathrm{CO}=\mathrm{SD} \times \mathrm{CSA} \times \mathrm{HR}
$$

where SD was stroke distance and CSA was the cross sectional area of left ventricular outflow tract.

The stroke distance was compared with stroke volume index to determine whether measurement of the cross sectional area was necessary $(\mathrm{SVI}=$ thermodilution $\mathrm{CO} /$ body surface area).

\section{ELECTRICAL BIOIMPEDANCE}

After skin preparation the bioimpedance monitor was connected to the patients via four pairs of low contact impedance electrodes. The lower thoracic voltage sensing electrodes were placed at the level of the xiphoid sternum in the midaxillary lines and the cervical sensing electrodes were positioned laterally at the base of the neck as close as possible to the clavicles. The "current injecting" electrodes delivered an alternating current at $70 \mathrm{kHz}$ and were placed with one pair $5 \mathrm{~cm}$ above the cervical sensing electrodes and the other pair $5 \mathrm{~cm}$ below the thoracic sensing electrodes. The BoMed NCCOM3 estimated stroke volume from the impedance signal recorded from the inner pairs of electrodes using the Stramek et al equation. $^{7}$

$$
S V=V E P T \times \text { VET } \times(\mathrm{dz} / \mathrm{dt}) \max / \mathrm{Z} 0
$$

Where VEPT $=$ volume of electrically participating tissue (a constant derived from body height and weight); VET = ventricular ejection time; $\mathrm{dz} / \mathrm{dt}=$ rate of change of impedance during systole; and $\mathrm{ZO}=$ basal thoracic impedance.

The monitor automatically averages stroke volume over twelve cardiac cycles and displays the cardiac output continuously. Electrical bioimpedance cardiac output was taken simultaneously with thermodilution measurements as the mean of 10 displayed values (that is the mean of 120 cardiac cycles). To investigate the accuracy of bioimpedance measurements without the influence of body size or heart rate, we also compared the stroke volume index measured by electrical bioimpedance with that measured by thermodilution. ${ }^{8}$

\section{REPRODUCIBILITY}

A pilot study was performed to measure the reproducibility of each non-invasive technique. Ten patients were studied who were all haemodynamically stable in the recovery phase after myocardial infarction. The bioimpedance monitor was applied as described above, and cross sectional echocardiography and Doppler studies were recorded on videotape. After 30 minutes' rest (to allow time for the electrode skin marks to disappear) the bioimpedance monitor was reapplied and a second cardiac output was recorded. Doppler echocardiography was performed a second time, by the same observer, and recorded on a separate videotape. The two tapes were analysed four weeks apart.

The coefficient of variation for repeat measurements by the same observer using electrical bioimpedance was $2 \cdot 7 \%$. The variability of echocardiographic measurement of aortic diameter was $1.5 \%$ and of the Doppler stroke distance $3.0 \%$. Thus the variability of Doppler echocardiographic measurements was greater than electrical bioimpedance, since the square of the aortic dimension is multiplied by the stroke distance in the calculation of cardiac output.

\section{STATISTICS}

Cardiac outputs measured by the different techniques were compared by the method of Bland and Altman." The intra-observer variability of the non-invasive methods was calculated as the coefficient of variation. Means were compared by Student's paired $t$ tests, and a $\mathrm{p}$ value $<0.05$ was regarded as significant. 


\section{Results \\ PATIENTS}

During the 10 month study, 29 patients had a Swan-Ganz catheter inserted within 24 hours of myocardial infarction. Four were excluded from the study because of aortic valve disease (two patients), severe obesity (one patient), or inadequate visualisation of left ventricular outflow tract on screening echocardiographic study (one patient). Therefore 25 patients (22 men), mean age 59 (range 47-72) years, were recruited and an attempt was made to measure cardiac output by all three techniques in every patient. This was successful in all but one patient, who was not suitable for Doppler echocardiographic examination (see below). The mean interval from the onset of pain to inclusion in the study was 16 hours 24 minutes. Acute myocardial infarction was confirmed subsequently by typical electrocardiographic changes and a rise in cardiac enzymes in all patients.

\section{DOPPLER CARDIAC OUTPUT VERSUS} THERMODILUTION

Cardiac output was measured by Doppler echocardiography in 24 patients; in one patient it was not possible to obtain a clear recording of aortic flow from either the apical or the suprasternal window. The mean (range) cardiac output by Doppler was $4.03(2.2-6.0) 1 / \mathrm{min}$, which was not significantly different from the mean cardiac output by thermodilution, 3.95 $(2 \cdot 1-6 \cdot 2) 1 / \min (p=0 \cdot 72)$

Analysis of the results by the method of Bland and Altman' (fig 2) gave the $95 \%$ limits of agreement as -1.23 to $+1.321 / \mathrm{min}(-31 \%$ to $+33 \%$ of the mean cardiac output). In three patients the Doppler result differed from thermodilution by more than $1 \mathrm{l} / \mathrm{min}$.

\section{ELECTRICAL BIOIMPEDANCE VERSUS}

\section{THERMODILUTION}

The mean (range) cardiac output by electical bioimpedance in 25 patients was $3.79(1 \cdot 1-6 \cdot 2)$ $1 / \mathrm{min}$, which did not differ significantly from thermodilution at $3.95(2 \cdot 1-6 \cdot 2) 1 / \mathrm{min}(\mathrm{p}=$ $0 \cdot 22$ ). The $95 \%$ limits of agreement (fig 3 ) were -1.43 to $+1 \cdot 111 / \mathrm{min}(-36 \%$ to $+28 \%$ of the mean cardiac output). In three patients the electrical bioimpedance result differed from thermodilution by more than $1 \mathrm{l} / \mathrm{min}$. Figure 2 Difference
between Doppler and thermodilution measurements of cardiac output plotted against the mean value.

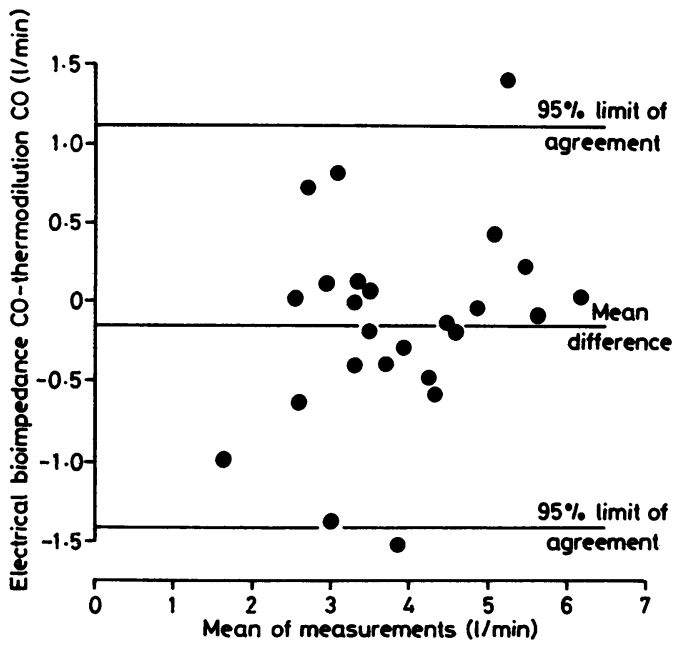

Figure 3 Difference between electrical bioimpedance and thermodilution measurements of cardiac output plotted against the mean value.

\section{DOPPLER AND ELECTRICAL BIOIMPEDANCE} MEASUREMENTS OF STROKE VOLUME INDEX The mean (range) stroke distance by Doppler was $14 \cdot 3(4 \cdot 3-21 \cdot 7) \mathrm{cm}$. When compared with thermodilution measurements of stroke volume index the correlation coefficient was $0.82 ; \mathrm{SD}=0.43 \times \mathrm{SVI}+3.38$. Stroke volume index was also calculated from the bioimpedance measurements. The $95 \%$ limits of agreement with thermodilution were $-8 \cdot 1$ to $+6 \cdot 7 \mathrm{ml} / \mathrm{m}^{2}$.

\section{Discussion}

To our knowledge this is the first reported study of the accuracy of electrical bioimpedance in patients with acute myocardial infarction, and only the second study of Doppler echocardiography. ${ }^{3}$ Previous reports compared Doppler determination of cardiac output with an invasive technique in other clinical settings. These studies, however, varied greatly in the numbers and types of patients studied, the number of observations per subject, the invasive technique used for comparison, and the Doppler method used. Good results were obtained with both pulsed and continuous wave Doppler, with sampling of flows in the aortic root, ascending aorta, descending aorta (including transoesophageal approach) and across the mitral, tricuspid, or pulmonary valves, ${ }^{10-12}$ but the results of different studies were variable. Three important studies compared the accuracy of these various Doppler sites in the same patients, and found the ascending aorta and left ventricular outflow methods to be the most accurate. ${ }^{5112}$ Similar comparisons showed that of the many aortic diameters that can be used in the calculations the aortic annulus or left ventricular outflow tract gives the best results, and when the ascending aortic root is used there is considerable overestimation of cardiac output. ${ }^{5613}$ It was possible to obtain measurements of aortic flow from either the suprasternal or apical window in $>90 \%$ of patients in a previous large study. ${ }^{11}$ We also found that this was the case in 24 of our 25 patients, and when both sites were success- 
fully studied the apical window gave the highest peak velocity in every case.

In the only previous study of Doppler measurements in acute myocardial infarction the methods used were comparable with our study, and the technique was feasible in all patients. It was also thought to be accurate, with a reported correlation coefficient of $0 \cdot 95$. $^{3}$ These workers, in common with most previous studies, investigated the agreement between the Doppler and invasive results solely by using the correlation coefficient. This is not a good method of determining the accuracy of a test or of comparing the results of one study with another. ${ }^{914}$ It can be considerably affected by a few reasonably accurate results at the extremes of a range of measurements and it is dependent upon the range under study (when cardiac output is studied the range depends upon the types of patients included). This may explain why studies in which cardiac outputs have varied by up to 20 fold have produced coefficients as high as $0.98 .{ }^{15} \mathrm{We}$ used the method of Bland and Altman to assess the accuracy of the technique. ${ }^{91416}$ This statistical method is not affected by the range under study or values at the extremes of the measurement, and it has the added advantage of expressing the results in a clinically meaningful manner. Using this method we showed that Doppler may overestimate the thermodilution cardiac output by up to $1.3 \mathrm{l} / \mathrm{min}$, or underestimate it by up to $1 \cdot 2 \mathrm{l} / \mathrm{min}$. Thus for $95 \%$ of measurements the thermodilution result would lie between $1.3 \mathrm{l} /$ min below and $1.21 / \mathrm{min}$ above a single Doppler estimate. The magnitude of these errors did not depend upon the absolute cardiac output, so they are potentially much more of a problem in patients with low cardiac outputs. For instance, a result of $3 \mathrm{l} / \mathrm{min}$ by either non-invasive technique may under or overestimate the thermodilution result by $37-47 \%$, whereas for a result of $6 \mathrm{l} / \mathrm{min}$ the error lies between $18 \%$ and $23 \%$.

Stroke distance measured by Doppler does not have the same units as stroke volume index measured by thermodilution, and because different variables were being compared we used regression analysis rather than the method of Bland and Altman. Regression analysis showed a close correlation $(r=0.82)$, which lends support to the adoption of stroke distance as a measure of cardiovascular function, as proposed by Haites et al. ${ }^{17}$ It is also important in practical terms because stroke distance can be measured by inexpensive "stand alone" Doppler equipment without the need for imaging. This is an interesting additional finding in a study designed primarily to investigate the measurement of cardiac output not stroke distance.

Interest has been aroused in electrical bioimpedance by the recent development of the new bioimpedance monitor (BoMed NCCOM 3 ) used in this study. ${ }^{819}$ A review of the published reports showed nine previous studies in adults in which this monitor was compared with thermodilution. All were conducted in intensive care, usually on patients after general surgery. Once again it is difficult to compare our results with previous reports because they have all relied on correlation coefficients to describe the accuracy of bioimpedance. None the less, our results seem to be broadly in keeping with previous experience. ${ }^{2021}$ We found that the cardiac output by thermodilution is likely to lie within a range from $1.1 \mathrm{l} / \mathrm{min}$ below to $1.41 / \mathrm{min}$ above a bioimpedance estimate. The high degree of reproducibility of electrical bioimpedance measurements in this study was also reported by others. ${ }^{19}$ In the largest series described so far, electrical bioimpedance could be measured in all of 58 critically ill patients after general surgery. ${ }^{22}$ Only one previous study has compared both Doppler and bioimpedance measurements with thermodilution, and this was conducted in an intensive care unit. ${ }^{20}$ Both techniques were feasible in $>85 \%$ of patients and, in keeping with our findings, bioimpedance was slightly more accurate than Doppler though the difference was not statistically significant.

Cardiac output is the product of stroke volume and heart rate and under normal conditions stroke volume is related to body surface area. Spurious associations can therefore be generated when cardiac output is calculated by a method, such as electrical bioimpedance, that incorporates both heart rate and body surface area in its formula. ${ }^{8}$ Indeed older bioimpedance monitors were unable reliably to determine stroke volume index, ${ }^{23}$ and estimated cardiac output mainly from the patient's size and heart rate. A recent study found that although cardiac output determined by the BoMed NCCOM3 correlated with indirect Fick measurements, this relation was no longer significant when stroke volume index was used instead of cardiac output. ${ }^{19}$ We therefore compared stroke volume index measured by electrical bioimpedance with thermodilution, and found that the $95 \%$ limits of agreement were $-8 \cdot 1$ to $+6.7 \mathrm{ml} / \mathrm{m}^{2}(-32 \cdot 4 \%$ to $+26 \cdot 8 \%$ of the mean stroke volume index). Thus electrical bioimpedance measurement of stroke volume index was just as accurate as the measurement of cardiac output. This study differs from the previous one, ${ }^{19}$ in which healthy volunteers were studied. Large changes in cardiac output were induced by maximal exercise such that a correlation was found between the two noninvasive techniques even though individual measurements varied widely. Because the increase in cardiac output that accompanies exercise is produced mainly by an increase in heart rate, ${ }^{24}$ a relatively smaller range of stroke volumes were studied. It is, therefore, not surprising that the correlation is less strong. A further problem with this study is that bioimpedance was compared with indirect Fick measurement of cardiac output rather than an invasive method. We used thermodilution as a "gold standard," and although we accept that this technique is far from perfect it is an established reference technique.

We found that both non-invasive techniques were feasible in patients with acute myocardial infarction. Doppler echocardiography required a lot of practice and the system we 
used took approximately 30 minutes to analyse each measurement. Electrical bioimpedance was highly reproducible, simple to use, and gave instant results. It has the additional advantage of allowing continuous monitoring of the cardiac output, which can be helpful in assessing the response to treatment.

1 George RJD, Winter RJD. The clinical value of measuring cardiac output. Br J Hosp Med 1985;34:89-95.

2 Forrester JS, Diamond G, McHugh TJ, Swan HJC. Filling Forrester JS, Diamond G, McHugh TJ, Swan HJC. Filling pressures in the right and left sides of the heart in acurt
myocardial infarction. N Engl J Med 1971;285:190-3

3 Mehta N, Bennett DE. Impaired left ventricular function in acute myocardial infarction assessed by Doppler measurement of ascending aortic blood velocity and maximal acceleration. Am J Cardiol 1986;57:1052-8.

4 Appel PJ, Kram HB, MacKabee J, Fleming AW, Shoemaker WC. Comparison of measurements of cardiac output by bioimpedance and thermodilution in severely ill surgical patients. Crit Care Med 1986;14:933-5.

5 Lewis JF, Kuo LC, Nelson JG, Limacher MC, Quinones MA. Pulsed Doppler echocardiographic determination of stroke volume and cardiac output: clinical validation of two methods using the apical window. Circulation 1984, 70:425-31.

6 Bouchard A, Blumlein S, Schiller NB, et al. Measurement of left ventricular stroke volume using continuous wave left ventricular stroke volume using continuous wave
Doppler echocardiography of the ascending aorta and $M$ mode echocardiography of the aortic valve. $\mathrm{J} \mathrm{Am} \mathrm{Coll}$ mode echocardiography

7 Stramek BB, Rose DM, Miyamoto A. Stroke volume equation with a linear base impedance and its accuracy, as compared to thermodilution and electromagnetic flow meter techniques in animals and humans. Proceedings of the sixth International Conference on Electrical Bioimpedance, Zadar, Yugoslavia. 1983:p38.

8 Smith SA. Pitfalls and limitations in the use of impedance cardiography [Letter]. Br Heart J 1989;61:129.

9 Bland JM, Altman DG. Statistical methods for assessing agreement between two methods of clinical measurement. Lancet 1986;i:307-10.

10 Zhang Y, Nitter-Hauge S, Ihlen H, Myhre E. Doppler echocardiographic measurement of cardiac output using the mitral orifice method. Br Heart J 1985;53:130-6.

11 Labovitz AJ, Buckingham TA, Habermehl K, Nelson J, Kennedy HL, Williams GA. The effects of sampling site on the two-dimensional echo-Doppler determination of cardiac output. Am Heart $J$ 1985;109:327-32.

12 Nicolosi GL, Pungercic E, Cervesato E, et al. Feasibility and variability of six methods for the echocardiographic and Doppler determination of cardiac output. Br Heart $J$ 1988;59:299-303.

13 Ihlen H, Amlie J, Dale J, et al. Determination of cardiac output by Doppler echocardiography. Br Heart J 1984;51: 54-60.

14 Diamond GA. Correlation, causation and agreement. $\mathrm{Am} \mathrm{J}$ Cardiol 1989;63:392.

15 Mehta N, Iyawe VI, Cummin AR, Bayley S, Saunders KB, Bennett ED. Validation of a Doppler technique for beatto-beat measurement of cardiac output. Clin Sci 1985; 69:-beat measu.

16 Ihlen H, Endresen K, Golf S, Nitter-Hauge S. Cardiac stroke volume during exercise measured by Doppler echocardiography: comparison with the thermodilution technique and evaluation of reproducibility. Br Heart $J$ 1987;58:455-9.

17 Haites NE, McLennan FM, Mowat DHR, Rawles JM. Assessment of cardiac output by the Doppler ultrasound technique alone. Br Heart J 1985;53:123-9.

18 Gibson DG. Stroke distance-an improved measure of cardiovascular function? $\mathrm{Br}$ Heart $J$ 1985;53:121-2.

19 Smith SA, Russell AE, West MJ, Chalmers J. Automated non-invasive measurement of cardiac output: comparison of electrical bioimpedance and carbon dioxide rebreathing techniques. Br Heart J 1988;59:292-8.

20 Wong DH, Bremper KD, Trujillo RJ, Zaccari J. Noninvasive cardiac output: simultaneous comparisons of two invasive cardiac output: simultaneous comparisons of two methods with

21 Shellock G, Jakubowski A. Measurement of cardiac output in critically ill patients by a new non-invasive bioimpedance system: comparison with the thermodilution methods [Abstract]. Physiologist 1987;30:21

22 Shoemaker WC, Appel PL, Kram HB, Nathan RC, Thompson JL. Multicomponent noninvasive physiologic monitoring of circulatory function. Crit Care Med 1988;16:482-90

23 Keim HJ, Wallace JM, Thurston H, Case DB, Drayer JIM, Laragh JH. Impedance cardiography for determination of stroke volume index. $J$ Appl Physiol 1976;41:797-800.

24 Chapman CB, Fisher JN, Sproule BJ. Behaviour of stroke volume at rest and during exercise in human beings. J Clin Invest 1960;39:1208-13. 